\title{
Epilepsias e Depressão
}

\section{RESUMO}

A associaçăo das epilepsias e da depressăo vem sendo abordada desde Hipócrates até os nossos dias. A presente revisăo enfoca estudos de prevaléncia da depressão interictal e dos aspectos relativos à ocorrẻncia de depressāo nos pacientes epilépticos.

\section{UNITERMOS}

Epilepsias Parciais. Depressåo
- Médica Pós-Graduanda da Disciplina de Neurologia. Setor de Investigação e Tratamento das Epilepsias (SITE)

- Chefe do SITE da UNIFESP-Escola Paulista de Medicina, SP, Brasil.

\author{
Ivette C. J. Kairalla * Carlos J. R. Campos"
}

A associação das epilepsias e da depressão, assim como seus estudos em separado, vem sendo abordada desde Hipócrates (460-375 a.C.), passando por Cardamus (séc. XVI) e Griesinger (séc. XVIII), até os nossos dias (Robertson, 1986). Alguns pacientes, quando recebem o diagnóstico de epilepsia, reagem com período de tristeza e de adaptação. Após elaborar esta situação, tendem a voltar ao humor normal. Há aqueles, contudo, que podem desenvolver um quadro depressivo com características de morbidade. Há ainda aqueles pacientes epilépticos em que depressão e ansiedade antecedem a primeira crise (Currie e col., 1971) e aqueles em que a depressão sucedeu o declínio da frequiência das crises (Betts, 1982).

Browne e Feldman (1983) sintetizam o conceito atual das epilepsias como sendo decorrentes de "uma descarga ocasional (paroxística), excessiva e desordenada dos neurônios, que pode ser detectada por manifestações clínicas, registros eletrencefalográficos (EEG) ou ambos". Estas descargas podem envolver uma parte do cérebro (epilepsias localizadas ou focais) ou uma área extensa atingindo os dois hemisférios (epilepsias generalizadas).

Quanto à depressão, clinicamente refere-se a uma diminuição das forças vitais que se expressa por uma redução dos sentimentos de prazer em relação a si mesmo, a alguém ou a algum aspecto da vida. Assim, é incluída tanto nos distúrbios da afetividade (função que diz respeito ao sentimento de inclinação para algo ou alguém) quanto nos do humor (função que se refere aos sentimentos de base que colorem a vida psíquica do indivíduo por si mesmo).

Do ponto de vista clínico, é importante identificarem-se os limites entre a depressão sem características de morbidade e aquela a nível de anormalidade que merece investigação clínica e tratamento. Considerando-se, do ponto de vista ontogenético, que um comportamento é adaptativo se ele promove o crescimento e sobrevivência dos membros individuais de uma espécie, pode-se dizer que a depressão, a nível mórbido, levaria o indivíduo a caminhar na direção contrária à preservação de si próprio e também da espécie.

Henri Ey (1969) aponta que, em psiquiatria, o termo "depressão", em caráter de morbidade, implica pelo menos 3 significados: pode se referir a um sintoma, a uma síndrome e também a uma entidade nosológica.

Não se conhece com exatidão a prevalência da depressão associada à epilepsia. Para Mendez e col. (1986) e Indaco e col. (1992), a prevalência 
de doença depressiva interictal em pacientes epilépticos é da ordem de 50 a $60 \%$ dos casos.

Carrieri e col. (1993) apontam as alterações de humor como sendo comuns na fase interictal das epilepsias, com frequiência predominante da depressão dos tipos major ou distímica, sendo raro o transtorno bipolar (Robertson e col., 1987; Indaco e col., 1992). Vários estudos registraram mais depressão em pacientes com crise parcial complexa se comparados com pacientes com outros tipos de crises (Dongier, 1959/1960; Hauser e col., 1993).

Considera-se o risco de suicídio no paciente epiléptico deprimido 4 a 5 vezes maior que na população em geral (Robertson, 1986; Mendez e col., 1986).

Segundo Robertson (1986), os desencadeantes da depressão interictal nas epilepsias incluem tanto fatores de risco neurobiológico como psicológicos. Contudo, na literatura há divergências com relação a este aspecto, talvez pela grande diversidade metodológica dos vários estudos (Indaco, 1992; Oxley, 1986). Discutem-se como fatores predisponentes à depressão no paciente epiléptico a frequiência das crises e a duração do quadro epiléptico (Robertson, 1989; Altshuler e col., 1990; Strauss e col., 1992), bem como a intensidade do tratamento e a idade de início da epilepsia e do tratamento (Reynolds \& Travers, 1974; Scott, 1977), a localização do foco epiléptico nas regiōes cerebrais (Altshuler e col., 1990; Hermann e col., 1991; Septien e col., 1993), a lateralização do foco (FlorHenry, 1969; Bear \& Fedio, 1977; Trimble \& Perez, 1980; Robinson e col., 1984; Indaco e col., 1992; Victoroff e col., 1994), efeitos colaterais das drogas antiepilépticas (Dodrill e Troupin, 1977; Indaco e col., 1992) e a influência do sexo (Flor-Henry, 1986; Kogeorgos e col., 1982; Septien e col., 1993).

Hermann e col. (1991) levantaram a hipótese de que os registros conflitantes da presença de depressão vinculada à lateralidade da lesão na epilepsia do lobo temporal podem ser devidos a alterações concomitantes do lobo frontal. Os lobos frontais estão intimamente envolvidos com funções integrativas superiores, como informação cognitiva processual. $O$ córtex pré-frontal em particular, que inclui a região frontal inferior, representa uma área de rica interconexão anatômica e funcional com várias outras regiões cerebrais, incluindo o córtex frontal dorsolateral, amígdala, hipotálamo, septo, tálamo medial e a formação reticular central do mesencéfalo.

A depressão no epiléptico parece se associar a distúrbios dos circuitos límbicos, seja de sua porção temporal (Altshuler e col., 1990), como também dos seus segmentos frontais, associação essa menos conhecida mas real, como pôde ser notado pela grande freqüência de síndromes depressivas associadas aos Acidentes Vasculares Cerebrais da parte anterior do lobo frontal esquerdo (Robinson e col., 1984).

A eletrocorticografia intra-operatória de pacientes a caminho de se submeter a lobectomia temporal anterior revela, com relativa freqüência, atividade epileptogênica nas regiões do lobo frontal, o que se supõe refletir uma parcela da atividade epileptiforme do foco de projeção temporal (Abou-Khalil e col., 1987; Hermann e col., 1988).

Bromfield e col. (1992) apontam o fato de que o substrato neurológico funcional não é necessariamente observado por técnicas de imagem anatômica. Em Acidente Vascular Cerebral, por exemplo, o hipometabolismo detectado através do PET-Scan pode ser observado longe da área infartada, presumivelmente refletindo áreas relacionadas sinapticamente. Contudo, estes autores relatam que na epilepsia parcial a área hipometabólica frequientemente se estende ao redor do foco identificado, um fenômeno que pode estar relacionado à existência de uma desaferentação funcional, atividade inibitória interictal, depleçāo metabólica pós-ictal ou outros mecanismos. A distribuição do hipometabolismo localizado também pode estar associada com estados interictais alterados de comportamento ou de cognição.

$\mathrm{O}$ achado mais comum na Positron Emission Tomography com F-2-deoxyglucose (FDG-PET) em pacientes com crises parciais, visto em 60 a $80 \%$ dos casos, é um metabolismo interictal reduzido ipsilateral ao foco eletrencefálico (Bromfield e col., 1992).

Em pacientes com descargas paroxísticas temporais mesiais nítidas, é geralmente observado hipometabolismo no córtex temporal lateral e freqüentemente no tálamo e lobo frontal. Embora as correlações clínicas de tais achados nāo sejam em geral bem entendidas, no caso do hipometabolismo frontal inferior seus dados sugerem uma possível associação entre fatores neuroquímicos e distúrbios psiquiátricos no paciente epiléptico.

A psicopatogenia da depressão, por sua vez, pode ser resumida em 3 teorias: 1) Teorias bioquímicas; 2) Teorias psicológicas; e 3) Teorias sociais (Guz, 1990). Destacamos, nesse caso, a condição humana chamada desamparo (Seligman, 1977) e a desesperança (Beck e col., 1974), bastante proeminentes no paciente epiléptico.

Considerando-se que os diversos fatores relacionados à associação das epilepsias e da depressão ainda não estão plenamente investigados, justifica-se o crescente interesse em relação à pesquisa nesta área, como mostra a literatura internacional. 
Mais do que apontar para assimetrias hemisféricas definidas, os achados dos diversos autores sugerem que avaliações de reações emocionais negativas graves devam resultar de abordagens mais amplas da situação global do paciente (Kurthen e col., 1991).

Lennox \& Markham (1953) exprimiram com precisão o nosso atual pensamento quando escreveram: "O bom médico se preocupa não somente com as ondas turbulentas do cérebro, mas com os distúrbios emocionais e com a injustiça social, pois o epiléptico não é apenas uma preparação neuro-muscular, ele é uma pessoa que apresenta uma combinação integrada do físico, mental, social e espiritual. Ruptura em qualquer uma das partes pode causar ou agravar a doença".

Apoio Financeiro: CNPq, CAPES, FINEP.

\section{Summary}

The association of epilepsies and depression has been approached since Hipocrates until nowadays. The present review gives emphasis to the studies of prevalence of interictal depression and the aspects related to the occurence of depression in the epileptics patients.

\section{Key words}

Partial Epilepsy. Depression

\section{Referências Bibliográfícas}

1. ABOU-KHALIL, B.W.; SIEGEL, G.J.; SACKELLARES, J.C.; GILMAN, S.; HICHWA, R.; MARSHALL, R. - Positron emission tomography studies of cerebral glucose metabolism in chronic partial epilepsy. Ann. Neurol. 22: 480-486, 1987.

2. ALTSHULER, L.L.; DEVINSKY, O.; POST, R.M.; THEODORE, W.H. - Depression, anxiety and temporal lobe epilepsy. Laterality of focus and symptoms. Arch. Neurol. 47(3): 284-288, 1990.

3. BEAR, D. \& FEDIO, P. - Quantitative analysis of interictal behavioral in temporal lobe epilepsy. Arch. Neurol. 34: 454-467, 1977.

4. BECK, A.T.; WEISSMAN, A.; LESTER, D.; TREXLER, L. - The measurement of pessimism: The hopelessness scale. J. Consult. Clin. Psychol. 42: 861-865, 1974.

5. BETTS, T.A. - Psychiatry and Epilepsy. In: LAIDLAW, J. \& RICKENS, A. - A textbook of Epilepsy. 2a. ed., Cap. 6. New York, Churchil \& Livingstone, 1982. p. 227-270.

6. BROMFIELD, E.B.; ALTSHULER, L.; LEIDERMAN, D.B.; BALISH, M.; KETTER, T.A.; DEVINSKY, O.; POST, R.M.; THEODORE, W.H. - Cerebral Metabolism and Depression in Patients with Complex Partial Seizures. Arch. Neurol. 49: 617. $623,1992$.

7. BROWNE, T.R. \& FELDMAN, R.G. - Epilepsy: An overview. In: Epilepsy - Diagnosis and Management. Cap. 1. Boston, Little, Brown and Company, 1983. p. 1-10.

8. CARRIERI, P.B.; PROVITERA, V; IACOVITTI, B.; IACHETTA, C.; NAPPI, C.; INDACO, A. - Mood disorders in epilepsy. Acta Neurologica 15(1): 62-67, 1993.
9. CURRIE, S.; HEATHFIELD, K.W.H.; HENSON, R.A.; SCOTT, D.F. - Clinical course and prognosis of temporal lobe epilepsy; a survey of 666 patients. Brain 94: 173-190, 1971.

10. DODRILL, C.B. \& TROUPIN, A.S. - Psychotropic effects of carbamazepine in epilepsy: A double-blind comparison with phenytoin. Neurology 27: 1023-1028, 1977.

11. DONGIER, S. - Statistical study of clinical and eletroencephalographic manifestations of 536 psychotic episodes occurring in 516 epileptics between clinical seizures. Epilepsia 1: 117-142, 1959-1960.

12. EY, H.; BERNARD, P.; BRISSET, C. - Estados Depresivos y crisis de melancolía. In: Tratado de Psiquiatría. Barcelona, TorayMason, S.A., 1969. p.237-72.

13. HAUSER, W.A.; ANNEGERS, J.F.; KURLAND, L.T. - Incidence of Epilepsy and Unprovoked Seizures in Rochester, Minnesota: 1935-1984. Epilepsia 34(3): 435-468, 1993.

14. FLOR-HENRY, P. - Psychosis and Temporal Lobe Epilepsy: a controlled investigation. Epilepsia 10:363-395, 1969.

15. FLOR-HENRY, P. - Observations, reflections and speculations on the cerebral determinants of mood and on the bilaterally asymmetrical distributions of the major neurotransmitter systems. Acta Neurol. Scand. 74(Suppl. 109): 75-89, 1986.

16. GUZ, I. - Teorias Comportamentais da Depressāo. In: Depressăo: $O$ que é, como se diagnostica e trata. Editora Roca Ltda., São Paulo, 1990. p.60.

17. HERMANN, B.P.; WYLER, A.R.; RICHEY, E.T. - Wisconsin Card Sorting Test performance in patients with complex partial seizures of temporal-lobe origin. J. Clin. Exp. Neuropsychol. 10: 46776, 1988.

18. HERMANN, B.P.; SEIDENBERG, M.; HALTINER, A.; WYLER, A.R. - Mood state in unilateral Temporal Lobe Epilepsy. Biol. Psychiatry 30: 1205-1218, 1991.

19. INDACO, A.; CARRIERI, P.B.; NAPPI, C.; GENTILE, S.; STRIANO, S. - Interictal depression in epilepsy. Epilepsy Research 12: 45-50, 1992.

20. KOGEORGOS, J.; FONAGY, P;; SCOTT, D.F. - Psychiatric symptom profiles of chronic epileptics attending a neurologic clinic: a controlled investigation. Br. J. Psychiat. 140: 236-243, 1982.

21. KURTHEN, M.; LINKE, D.B.; REUTER, B.M.; HUFNAGEL, A.; ELGER, C.E. - Severe negative emotional reactions in intracarotid sodium amytal procedures: further evidence for hemispheric asymmetrics. Cortex 27(2): 333-337, 1991.

22. LENNOX, W.G. \& MARKHAN, C.H. - The socio-psychological treatment of epilepsy. JAMA 152: 1690-1694, 1953.

23. MENDEZ, M.F.; CUMMINGS, J.L.; BENSON, D.F. - Depression in epilepsy: significance and phenomenology. Arch. Neurol. 43: 766-770, 1986.

24. OXLEY, J.; SANDER, JWAS.; THOMPSON, PJ. - Epilepsy as a chronic handicap. In: TRIMBLE, MR. \& BOLWIG, TG. - Aspects of Epilepsy and Psychiatry. Great Britain, John Wiley \& Sons, 1986. p. 235-46.

25. REYNOLDS, E.H, \& TRAVERS, R.D. - Serum anticonvulsant concentrations in epileptic patients with mental symptoms. Br. J. Psychiat. 124: 440-445, 1974.

26. ROBERTSON, M.M. - Ictal and interictal depression in patients with epilepsy. In: TRIMBLE, M.R. \& BOLWIG, T.G. - Aspects of Epilepsy and Psychiatry. Great Britain, John Wiley \& Sons, 1986. p. 213-33.

27. ROBERTSON, M.M.; TRIMBLE, M.R.; TOWNSEND, H.R.A. Phenomenology of depression in epilepsy. Epilepsia 28(4): 364372, 1987.

28. ROBERTSON, M.M. - The organic contribution to depressive illness in patients with epilepsy. J. Epilepsy, 2: 189-230, 1989. 
29. ROBINSON, R.G.; KUBOS, K.L.; STARR, L.B.; RAO, K.; PRICE, T.R. - Mood disorders in stroke patients: importance of location of lesion. Brain 107: 81-93, 1984.

30. SCOTT, D.F. - Psychiatric aspects of epilepsy. Br. J. Psychiat. 132: 417-430, 1977.

31. SELIGMAN, M.E.P. - Desamparo - Sobre Depressão, Desenvolvimento e Morte. Tradução Maria Teresa de Araújo Silva. Ed. de Humanismo, Ciências e Tecnologia "HUCITEC" Ltda. Editora da Universidade de São Paulo, São Paulo - Br. 1977. 223 p.

32. SEPTIEN, L.; GRAS, P.; GIROUD, M.; DIDI-ROY, R.; BRUNOTTE, F.; PELLETIER, J.L.; DUMAS, R. - Dépression et épilepsie temporale. Rôle possible de la latéralité du foyer épileptique et du sexe. Neurophysiol. Clin. 23: 327-335, 1993.

33. STRAuSS, E.; WADA, J.; MOLL, A. - Depression in male and female subjects with complex partial seizures. Arch. Neurol. 49(4): 391-392, 1992.

34. TRIMBLE, M.R.; PEREZ, M.M. - Quantification of psychopathology in adult patients with epilepsy. In: KULING, B.M.; MEINARDI, H.; STORES, G. - Epilepsy and Behavior. Lisse: Swets and Zeitlinger, 1980. p. 118-26.

35. VICTOAOFF, J.I.; BENSON, D.F.; GRAFTON, S.T.; ENGEL Jr., J.; MAZZIOTTA, J.C. - Depression in Complex Partial Seizures. Arch. Neurol. 51: 155-63, 1994. 